\title{
Evolution of electron spectrum during March 2012 by ARINA spectrometer data
}

Aleksandrin S.Yu., ${ }^{a}$ Galper A.M., ${ }^{a}$ Mikhailov V.V. ${ }^{a, *}$ and Zharaspayev T.R. ${ }^{a}$

${ }^{a}$ National Research Nuclear University MEPhI (Moscow Engineering Physics Institute) 115409, Russian Federation, Moscow, Kashirskoe shosse, 31

E-mail: syaleksandrin@mephi.ru, VVMikhajlov@mephi.ru

Electron fluxes with energies of 3-30 MeV were analyzed using data from the ARINA satellite experiment. The changes in the spectrum of high-energy electrons in March 2012 were analyzed.

$37^{\text {th }}$ International Cosmic Ray Conference (ICRC 2021)

July 12th-23rd, 2021

Online - Berlin, Germany

*Presenter 


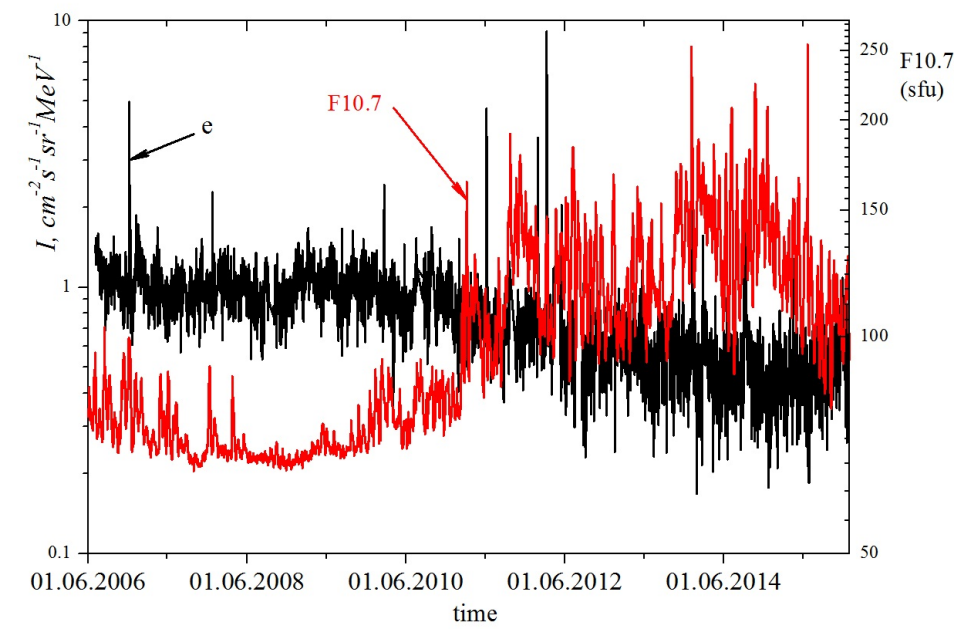

Figure 1: Solar activity index [1] and electron flux (ARINA data, E=3-30 MeV)

\section{Introduction}

Galactic cosmic ray (GCR) variations have been studied for a long time and are of great interest. They can be periodic, associated with solar cycles, and short-term, caused by solar flares, coronal mass ejection (CME). GCR variations changes are observed on ground-based installations - neutron monitors, muon telescopes, and in space experiments. In this work, we used data from the ARINA space experiment launched in 2006 and operating in orbit for almost 10 years during the end of the $23 \mathrm{rd}$ and the beginning of 24 solar cycles [2],[3]. The inclination was about $70^{\circ}$. The device measured the flux and energy of electrons with energies from 3 to $30 \mathrm{MeV}$ and protons from 30 to $100 \mathrm{MeV}$.

\section{High-energy electron flux in L-shell above 9}

GCR fluxes are stable in quiet solar activity, but long-term variations are observed associated with changes in solar activity during an quasi-periodic 11-year cycle (Figure 1). Here we have considered flux of relativistic electrons. To select GCR electrons, flux measurements were selected when the L-shell was greater than 9. The figure shows the electron flux with energies in the range of 3-5 MeV for the entire observation period by the spectrometer (black curve). The red curve shows the solar radio flux at $10.7 \mathrm{~cm}(2800 \mathrm{MHz})$, which characterizes solar activity. It can be seen that the flux of such electron changes approximately twice during the solar cycle; the minimum is observed during the maximum solar activity in 2014.

In addition, there are short-term changes in fluxes, which can increase by 10 times or more. This change took place in March 2012. This work is devoted to the study of this event.

The following figure (Figure 2) shows the proton and electron fluxes in March 2012. It can be seen that there was a sharp increase in the fluxes of both protons and electrons on March 7, which then went down. On March 7, a X5.4-class solar flare occurred in the sun (on March 7 at 00:28 UTC, SunSpot 1429, CME [4]). An increase in electron fluxes was observed on many spacecraft GOES, Stereo-A, B [5], [6]. 


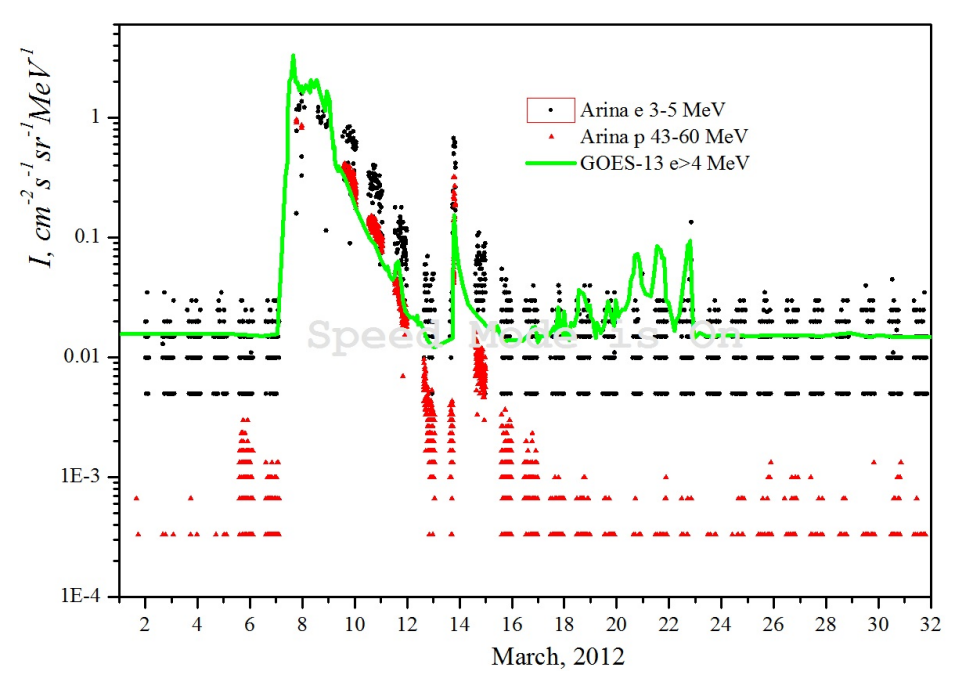

Figure 2: Electron flux by ARINA and GOES-13 [7] data on Match 2012

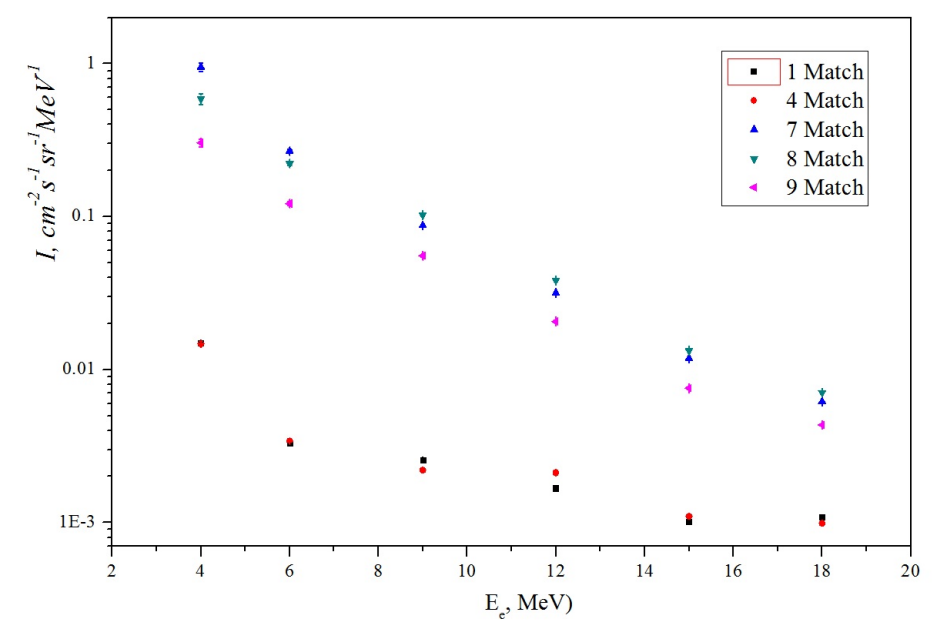

Figure 3: Electron flux spectrum during solar flare on 7 Match 2012 by ARINA

The ARINA spectrometer made it possible to measure the energy of electrons. Below is the spectrum of the flux of registered electrons before the solar flare and immediately after.

The spectra of March 1 and 4 correspond to the quiet period before the flare (the spectrum has the same form after the end of the disturbance on March 20), during the active phase of the flare, the electron flux increases several tens of times and the spectrum becomes softer (Figure 3). The spectrum index in quiet periods is $\gamma \approx 2$, during the solar flare $\gamma \approx 4$.

\section{Conclusion}

The high-energy electron flux registered by the ARINA spectrometer on large L-shells ( $\mathrm{L}>9)$ in March 2012 during a solar flare (SunSpot 1429, X5.4) was studied. The flux had increased more than 10 times. The spectrum of electrons of the solar flare was softer $(\gamma \approx 4)$ relative to galactic electrons $(\gamma \approx 2)$, which are recorded during quiet periods of observation. 


\section{References}

[1] https://omniweb.gsfc.nasa.gov/form/dx1.html

[2] A.V. Bakaldin, A.G. Batischev, A.M. Galper et al. Cosmic Research. Vol 45. No 5. p. 471 (2007)

[3] S. Yu. Aleksandrin, A. M. Galper, T. R. Zharaspayev, and S. V. Koldashov Bulletin of the Russian Academy of Sciences: Physics, 2015 Vol. 79, No. 5, pp. 646-648.

[4] https://www.spaceweatherlive.com/en/archive/2012/03/07/xray.html

[5] https://www.nesdis.noaa.gov/content/goes-13-turns-10

[6] https://solarsystem.nasa.gov/missions/stereo/in-depth/

[7] https://satdat.ngdc.noaa.gov/sem/goes/data/avg/2012/03/ 